University of Wollongong

Research Online

Faculty of Engineering and Information

Faculty of Engineering and Information

Sciences - Papers: Part B

Sciences

2019

A semianalytical formula for European options under a hybrid Heston-CoxIngersoll-Ross model with regime switching

Xinjiang $\mathrm{He}$

University of Wollongong, xinjiang@uow.edu.au

Wenting Chen

Jiangnan University, wtchen@uow.edu.au

Follow this and additional works at: https://ro.uow.edu.au/eispapers1

Part of the Engineering Commons, and the Science and Technology Studies Commons

Research Online is the open access institutional repository for the University of Wollongong. For further information contact the UOW Library: research-pubs@uow.edu.au 


\title{
A semianalytical formula for European options under a hybrid Heston-Cox- Ingersoll-Ross model with regime switching
}

\author{
Abstract \\ In this paper, we consider the pricing of European options under a regime-switching Heston-Cox-Ingersoll- \\ Ross (CIR) hybrid model, where the mean-reversion levels of both the stochastic volatility and interest rate \\ are assumed to change among different states. Albeit difficult, we have still managed to derive an \\ semianalytical pricing formula for European options after the generalized moment generating function of \\ this particular model is worked out. Numerical experiments are also carried out to demonstrate the \\ accuracy of the newly derived formula as well as the influence of the introduction of the regime-switching \\ mechanics on option prices. Finally, through a preliminary empirical study, our model is shown to be \\ superior to the Heston-CIR model, which demonstrates the importance of introducing the regime- \\ switching mechanics.

\section{Disciplines} \\ Engineering | Science and Technology Studies

\section{Publication Details} \\ He, X. \& Chen, W. (2019). A semianalytical formula for European options under a hybrid Heston-Cox- \\ Ingersoll-Ross model with regime switching. International Journal of Finance and Economics, Online First \\ 1-10.
}




\title{
A semi-analytical formula for European options under a hybrid Heston-CIR model with regime switching
}

\author{
Xin-Jiang $\mathrm{HE}^{*} \quad$ Wenting Chen ${ }^{\dagger}$
}

\begin{abstract}
In this paper, we consider the pricing of European options under a regime switching Heston-CIR hybrid model, where the mean-reversion levels of both the stochastic volatility and interest rate are assumed to change among different states. Albeit difficult, we have still managed to derive an semi-analytical pricing formula for European options after the generalized moment generating function of this particular model is worked out. Numerical experiments are also carried out to demonstrate the accuracy of the newly derived formula as well as the influence of the introduction of the regime switching mechanics on option prices. Finally, through a preliminary empirical study, our model is shown to be superior to the Heston-CIR model, which demonstrates the importance of introducing the regime switching mechanics.
\end{abstract}

\section{AMS(MOS) subject classification.}

Keywords. Heston-CIR hybrid model, regime switching, generalized moment generating function, semi-analytical solution, European options, empirical study.

\footnotetext{
*School of Mathematics and Applied Statistics, University of Wollongong NSW 2522, Australia

${ }^{\dagger}$ Corresponding author. Department of Business, Jiangnan University, Jiangsu, China.
} 


\section{Introduction}

It is nowadays widely acknowledged that the well-known Black-Scholes model (Black and Scholes, 1973), which assumes that the underlying price follows a geometric Brownian motion, is not adequate in pricing financial derivatives. Some of its assumptions made to achieve analytical simplicity and tractability are not appropriate and at odds with financial market observations. A typical example is the so-called "volatility smile" phenomenon (Dumas et al., 1998), which suggests that the implied volatility extracted from real financial data tends to exhibit a "smile" curve instead of a flat line, demonstrating the inappropriateness of the constant volatility assumption. Therefore, a number of modifications have been proposed to deal with the drawbacks of the Black-Scholes model. Among them, the stochastic volatility has received the most attention.

Unfortunately, it is usually difficult to find analytical solutions even for the price of European options when the volatility is assumed to be governed by another stochastic source. In this case, numerical methods have been widely used in dealing with derivative pricing problems. For instance, the Monte-Carlo simulation technique was adopted by Johnson and Shanno (1987) and Scott (1987) to price options when the variance is assumed to be changing, while the finite difference method was employed in (Wiggins, 1987) to solve the PDEs (partial differential equations) governing option prices under stochastic volatility models. However, it needs to be pointed out that there are two main disadvantages in using numerical methods. One is that some systematic errors are often induced by numerical methods, resulting in inaccurate results. The other is that model calibration, a process that any mathematical model needs to go through before it can be safely applied to real financial markets, is usually time-intensive, and numerical methods, often requiring a fair bit of time to be implemented, may even worsen the situation. Given the drawbacks associated with the numerical methods, a number of authors have been concentrating on finding analytical solutions under stochastic volatility models. 
In the literature, Hull and White (1987) managed to provide a power series solution for European option prices when the volatility is modeled by another geometric Brownian motion. Stein and Stein (1991) derived an analytical pricing formula for European options when the volatility follows an Ornstein-Uhlenbeck process. However, both models suffer from some model flaws, affecting their potential applications in practice. For example, the zero correlation assumption between the underlying price and volatility under the Hull-White model contradicts the so-called "leverage effects", which demonstrate that the underlying price and the volatility should be negatively correlated with each other (Bakshi et al., 1997). The Stein-Stein model is unable to prevent the volatility from dropping below zero, which is certainly unrealistic. A breakthrough took place in 1993, when Heston (1993) proposed to describe the volatility with the CIR (Cox-Ingersoll-Ross) process. The Heston model has been proved to satisfy a range of basic properties, including the volatility being non-negative and mean-reverting (Beckers, 1983). Under this model, a closed-form pricing formula for European options can also be derived, which enables its calibration to be conducted in a reasonable speed. Moreover, He and Zhu (2016b) and Zhu and He (2017) went even further and provided a different analytical pricing formula for European options under this particular model by choosing the minimal entropy martingale measure, as a result of the market being incomplete after the introduction of the stochastic volatility.

However, the Heston model is still unable to perfectly describe the dynamics of the underlying price, and a large amount of researchers are still working on finding more appropriate models that are closer to reality. A natural modification to the Heston model is to add time-dependent parameters to the volatility process to provide more flexibility (Forde and Jacquier, 2010). Another popular choice is to assume that the interest rate under the Heston model also be stochastic. Such kind of hybrid models combining the Heston model and some stochastic interest rate models are useful particularly for pricing financial derivatives (Abudy and Izhakian, 2013; Grzelak and Oosterlee, 2011; He and Zhu, 2018a). Recently, lots of empirical evidence suggests the existence of regime switching 
in real financial markets (Eraker, 2004; Hamilton, 1990), which prompts the adoption of different Markov-modulated models in pricing financial derivatives (Buffington and Elliott, 2002; He and Zhu, 2017, 2018b). It is even pointed out by Vo (2009) that regime-switching stochastic volatility models can not only enhance the forecasting power of the stochastic volatility models, but also better capture major events affecting the market. Therefore, regime switching Heston models (Elliott and Lian, 2013; He and Zhu, 2016a) have also drawn attentions from both academic researchers and market practitioners.

In this paper, we adopt a regime switching Heston-CIR hybrid model. In this model, both the volatility and interest rate are assumed to be stochastic, with the mean-reversion levels of both the volatility and interest rate changing among different states. Because of the complexity of the model, it is quite difficult to solve the prices of financial derivatives either numerically or analytically. As pointed out in (Cao et al., 2018), there exists a gap in the literature regarding pricing financial derivatives under stochastic volatility and interest rate with regime-switching. Albeit difficult, we have managed to derive a semi-analytical pricing formula for European options after the successful derivation of the exact form of the generalized moment generating function. Numerical experiments are also carried out to show the accuracy of our formula as well as the effect of the newly introduced regime switching factor. Finally, an empirical study is conducted to compare our model and the original Heston-CIR model.

The rest of the paper is organized as follows. In Section 2, the adopted regime switching Heston-CIR hybrid model is briefly introduced. In Section 3, the solution procedure is illustrated in details. In Section 4, numerical experiments are carried out to show various properties of the newly derived formula. In Section 5, empirical results are presented to show the importance of introducing regime switching into the Heston-CIR model, followed by some concluding remarks in the last section. 


\section{The regime switching Heston-CIR hybrid model}

In this section, the Heston-CIR hybrid model, with both the stochastic volatility and interest rate being governed by different CIR processes, is combined with regime switching mechanics by allowing the mean-reversion levels of both the volatility and the interest rate to jump between different states following a Markov chain, in order to account for the effect of regime switching on the option prices. With the underlying price, volatility and interest rate being respectively represented by $S_{t}, v_{t}$ and $r_{t}$, the specific model dynamics under a risk-neutral measure $\mathbb{Q}$ can be expressed as

$$
\begin{aligned}
\frac{d S_{t}}{S_{t}} & =r_{t} d t+\sqrt{v_{t}} d W_{t}^{S} \\
d v_{t} & =k\left(\theta_{X_{t}}-v_{t}\right) d t+\sigma \sqrt{v_{t}} d W_{t}^{v} \\
d r_{t} & =\alpha\left(\beta_{X_{t}}-r_{t}\right) d t+\eta \sqrt{r_{t}} d W_{t}^{r}
\end{aligned}
$$

Here, $W_{t}^{S}$ and $W_{t}^{v}$ are two standard Brownian motions with correlation $\rho$, while $W_{t}^{r}$ is another Brownian motion being independent with the other two. $X_{t}$ is a continuous time Markov chain, independent of the three Brownian motions, being defined as ${ }^{1}$

$$
X_{t}= \begin{cases}(1,0)^{T}, & \text { when the economy is believed to be in State 1 } \\ (0,1)^{T}, & \text { when the economy is believed to be in State } 2\end{cases}
$$

The transition between the two states follows a Poisson process as

$$
P\left(t_{i j}>t\right)=e^{-\lambda_{i j} t}, i, j=1,2, i \neq j
$$

with $\lambda_{i j}$ and $t_{i j}$ being the transition rate from State $i$ to $j$ and the time spent in State $i$ before transferring to State $j$, respectively. In this case, the mean-reversion levels of the

\footnotetext{
${ }^{1}$ In our discussion, we will focus on the two-state Markov chain, and the extension to arbitrary but finite states is straightforward.
} 
volatility and interest rate can be derived through

$$
\theta_{X_{t}}=<\bar{\theta}, X_{t}>, \quad \beta_{X_{t}}=<\bar{\beta}, X_{t}>,
$$

with $\bar{\theta}=\left(\theta_{1}, \theta_{2}\right)^{T}, \bar{\beta}=\left(\beta_{1}, \beta_{2}\right)^{T}$, and $<\cdot, \cdot>$ denoting the inner product of two vectors.

To facilitate the process of deriving the European option pricing formula in the next section, the model dynamics are alternatively expressed as

$$
d Y_{t}=\mu\left(Y_{t}\right) d t+\Sigma\left(Y_{t}\right) d W_{t},
$$

where $Y_{t}$ and $W_{t}$ are defined as

$$
Y_{t}=\left[\begin{array}{c}
z_{t} \\
v_{t} \\
r_{t}
\end{array}\right], \quad W_{t}=\left[\begin{array}{c}
W_{1, t} \\
W_{2, t} \\
W_{3, t}
\end{array}\right]
$$

with $z_{t}=\ln \left(S_{t}\right)$, and the three Brownian motions, $W_{1, t}, W_{2, t}$ and $W_{3, t}$, being independent with each other. The drift term $\mu\left(Y_{t}\right)$ here can be specified as

$$
\mu\left(Y_{t}\right)=\left[\begin{array}{c}
r_{t}-\frac{1}{2} v_{t} \\
k\left(\theta_{X_{t}}-v_{t}\right) \\
\alpha\left(\beta_{X_{t}}-r_{t}\right)
\end{array}\right]=K_{0}+K_{1} Y_{t}
$$

with

$$
K_{0}=\left[\begin{array}{c}
0 \\
k \theta_{X_{t}} \\
\alpha \beta_{X_{t}}
\end{array}\right], \quad K_{1}=\left[\begin{array}{ccc}
0 & -\frac{1}{2} & 0 \\
0 & -k & 0 \\
0 & 0 & -\alpha
\end{array}\right] \text {, }
$$


and the expression of the volatility term $\Sigma\left(Y_{t}\right)$ is given as

$$
\Sigma\left(Y_{t}\right)=\left[\begin{array}{ccc}
\sqrt{v_{t}} & 0 & 0 \\
\sigma \rho \sqrt{v_{t}} & \sigma \sqrt{\left(1-\rho^{2}\right) v_{t}} & 0 \\
0 & 0 & \eta \sqrt{r_{t}}
\end{array}\right] .
$$

With the dynamics of the regime switching Heston-CIR hybrid model being presented, the determination of European option prices under this particular model will be discussed in the following section.

\section{Pricing European options}

This section is divided into two subsections. In the first subsection, the general approach for the pricing of European options is presented, with the only unknown part of the pricing formula being the generalized moment generating function. In the second subsection, this unknown function is worked out explicitly, with which the target pricing formula is then successfully derived.

\subsection{The general pricing approach}

We begin by showing that the SDE system (2.2) is affine. As we have already shown in Equation (2.4) that the drift term $\mu\left(Y_{t}\right)$ can be expressed as an affine function, the left task is to transform both $\Sigma\left(Y_{t}\right) \Sigma^{T}\left(Y_{t}\right)$ and the discounted factor $R\left(Y_{t}\right)$ into affine functions.

In particular, $\Sigma\left(Y_{t}\right) \Sigma^{T}\left(Y_{t}\right)$ can be formulated as

$$
\Sigma\left(Y_{t}\right) \Sigma^{T}\left(Y_{t}\right)=\left[\begin{array}{ccc}
v_{t} & \rho \sigma v_{t} & 0 \\
\rho \sigma v_{t} & \sigma^{2} v_{t} & 0 \\
0 & 0 & \eta^{2} r_{t}
\end{array}\right]=H \cdot Y_{t}
$$


where $H$ is a $3 \times 3 \times 3$ matrix, whose entries, $h_{i j}, 1 \leq i, j \leq 3$, are $3 \times 1$ vectors, specified as

$$
h_{11}=\left[\begin{array}{c}
0 \\
1 \\
0
\end{array}\right], h_{22}=\left[\begin{array}{c}
0 \\
\sigma^{2} \\
0
\end{array}\right], h_{33}=\left[\begin{array}{c}
0 \\
0 \\
\eta^{2}
\end{array}\right]
$$

and

$$
h_{12}=h_{21}=\left[\begin{array}{c}
0 \\
\rho \sigma \\
0
\end{array}\right], \quad h_{13}=h_{23}=h_{31}=h_{32}=\left[\begin{array}{l}
0 \\
0 \\
0
\end{array}\right] \text {. }
$$

On the other hand, the discounted factor $R\left(Y_{t}\right)$ is the risk-free interest rate $r_{t}$, and thus, it can be written as

$$
R\left(Y_{t}\right)=\epsilon_{3} \cdot Y_{t}
$$

where $\epsilon_{3}=(0,0,1)^{T}$. Combining (2.4), (3.1) and (3.2), we can reach a conclusion that (2.2) is affine.

Bearing this in mind, the European option prices denoted by $U\left(Y_{t}, X_{t}, t\right)$ can now be determined from ${ }^{2}$

$$
\begin{aligned}
U\left(Y_{t}, X_{t}, t\right) & =E\left[e^{-\int_{t}^{T} R\left(Y_{s}\right) d s}\left(S_{T}-K\right)^{+} \mid Y_{t}, X_{t}\right] \\
& =E\left[e^{-\int_{t}^{T} R\left(Y_{s}\right) d s}\left(e^{z_{T}}-K\right)^{+} \mid Y_{t}, X_{t}\right] \\
& =E\left[e^{-\int_{t}^{T} R\left(Y_{s}\right) d s}\left(e^{\epsilon_{1} \cdot Y_{t}}-K\right)^{+} \mid Y_{t}, X_{t}\right] \\
& =G_{\epsilon_{1},-\epsilon_{1}}\left(-\ln (K) ; Y_{t}, X_{t}, t, T\right)-K G_{0,-\epsilon_{1}}\left(-\ln (K) ; Y_{t}, X_{t}, t, T\right)
\end{aligned}
$$

\footnotetext{
${ }^{2}$ We refer interested readers to (Duffie et al., 2000) for the details on the derivation of this general pricing formula.
} 
with $\epsilon_{1}=(1,0,0)^{T}$, and

$$
G_{a, b}\left(c ; Y_{t}, X_{t}, t, T\right)=\frac{f\left(a, Y_{t}, X_{t}, t, T\right)}{2}-\frac{1}{\pi} \int_{0}^{+\infty} \frac{\operatorname{Im}\left[f\left(a+j u b, Y_{t}, X_{t}, t, T\right) e^{-j u c}\right]}{u} d u
$$

where $j$ is the imaginary unit, and $\operatorname{Im}(\cdot)$ demotes the imaginary part. $f\left(\phi, Y_{t}, X_{t}, t, T\right)$ is the generalized moment generating function defined as

$$
f\left(\phi, Y_{t}, X_{t}, t, T\right)=E\left[e^{-\int_{t}^{T} R\left(Y_{s}\right) d s} \cdot e^{\phi \cdot Y_{T}} \mid Y_{t}, X_{t}\right]
$$

where $\phi=\left(\phi_{1}, \phi_{2}, \phi_{3}\right)^{T}$.

Clearly, the only unknown term in the general pricing formula (3.3), is the generalized moment generating function $f$, and the details on its derivation will be provided in the next subsection.

\subsection{The generalized moment generating function}

Due to the existence of the Markov chain, the direct computation of the expectation involved in (3.5) is extremely difficult. To facilitate the computation, we rearrange the generalized moment generating function $f\left(\phi, Y_{t}, X_{t}, t, T\right)$ as

$$
f\left(\phi, Y_{t}, X_{t}, t, T\right)=E\left\{E\left[e^{-\int_{t}^{T} R\left(Y_{s}\right) d s} \cdot e^{\phi \cdot Y_{T}} \mid Y_{t}, X_{T}\right] \mid X_{t}\right\}
$$

In other words, we shall firstly work out the inner expectation, before we are able to

derive the generalized moment generating function. If we define $m\left(\phi, Y_{t}, t, T \mid X_{T}\right)$ as the generalized moment generating function conditional upon all the information of the Markov chain up to the expiry time, i.e.,

$$
m\left(\phi, Y_{t}, t, T \mid X_{T}\right)=E\left[e^{-\int_{t}^{T} R\left(Y_{s}\right) d s} \cdot e^{\phi \cdot Y_{T}} \mid Y_{t}, X_{T}\right]
$$


its expression can be explicitly derived and is provided in the following theorem.

Theorem 1. If the dynamics of the underlying price $S_{t}$, volatility $v_{t}$ and interest rate $r_{t}$ are as given in Equation (2.2), the conditional generalized moment generating function $m\left(\phi, Y_{t}, t, T \mid X_{T}\right)$ can be specified as

$$
m=e^{C(\phi ; \tau)+D(\phi ; \tau) \cdot Y_{t}}
$$

with $\cdot$ being the dot product for vectors, $\tau=T-t, D(\phi ; \tau)=\left[\begin{array}{c}D_{1}(\phi ; \tau) \\ D_{2}(\phi ; \tau) \\ D_{3}(\phi ; \tau)\end{array}\right]$, and

$$
\begin{aligned}
D_{1}(\phi ; \tau) & =\phi_{1}, \\
D_{2}(\phi ; \tau) & =\frac{d_{1}-\left(\rho \sigma \phi_{1}+\sigma^{2} \phi_{2}-k\right)}{\sigma^{2}} \cdot \frac{1-e^{d_{1} \tau}}{1-g_{1} e^{d_{1} \tau}}+\phi_{2}, \\
D_{3}(\phi ; \tau) & =\frac{d_{2}-\left(\eta^{2} \phi_{3}-\alpha\right)}{\eta^{2}} \cdot \frac{1-e^{d_{2} \tau}}{1-g_{2} e^{d_{2} \tau}}+\phi_{3}, \\
C(\phi ; \tau) & =k \int_{t}^{T}<\bar{\theta}, X_{s}>D_{2}(\phi ; T-s) d s+\alpha \int_{t}^{T}<\bar{\beta}, X_{s}>D_{3}(\phi ; T-s) d s, \\
d_{1} & =\sqrt{\left(\rho \sigma \phi_{1}+\sigma^{2} \phi_{2}-k\right)^{2}+\sigma^{2}\left(\phi_{1}-\phi_{1}^{2}+2 k \phi_{2}-2 \rho \sigma \phi_{1} \phi_{2}-\sigma^{2} \phi_{2}^{2}\right)}, \\
d_{2} & =\sqrt{\left(\eta^{2} \phi_{3}-\alpha\right)^{2}+\eta^{2}\left(2 \alpha \phi_{2}+2-2 \phi_{1}-\eta^{2} \phi_{3}^{2}\right)}, \\
g_{1} & =\frac{\left(\rho \sigma \phi_{1}+\sigma^{2} \phi_{2}-k\right)-d_{1}}{\left(\rho \sigma \phi_{1}+\sigma^{2} \phi_{2}-k\right)+d_{1}}, g_{2}=\frac{\left(\eta^{2} \phi_{3}-\alpha\right)-d_{2}}{\left(\eta^{2} \phi_{3}-\alpha\right)+d_{2}} .
\end{aligned}
$$

Proof. According to the properties of the affine jump diffusions, as shown in (Duffie et al., 2000), it is not difficult to find that the conditional generalized moment generating function $m\left(\phi, Y_{t}, t, T \mid X_{T}\right)$ defined in (3.7) can be written in the form of (3.8), with the involved functions satisfying the following coupled ODE system

$$
\begin{aligned}
\frac{d}{d \tau} D(\phi ; \tau) & =K_{1}^{T} D(\phi ; \tau)+\frac{1}{2} D^{T}(\phi ; \tau) H D(\phi ; \tau)-\epsilon_{3}, D(\phi ; 0)=\phi, \\
\frac{d}{d \tau} C(\phi ; \tau) & =K_{0} \cdot D(\phi ; \tau), C(\phi ; 0)=0 .
\end{aligned}
$$


According to the definition of $D(\phi ; \tau)$, we have

$$
\begin{aligned}
\frac{d}{d \tau} D_{1} & =0, D_{1}(\phi ; 0)=\phi_{1} \\
\frac{d}{d \tau} D_{2} & =\frac{1}{2} \sigma^{2} D_{2}^{2}+\left(\rho \sigma D_{1}-k\right) D_{2}-\frac{1}{2}\left(D_{1}-D_{1}^{2}\right), D_{2}(\phi ; 0)=\phi_{2}, \\
\frac{d}{d \tau} D_{3} & =\frac{1}{2} \eta^{2} D_{3}^{2}-\alpha D_{3}+D_{1}-1, D_{3}(\phi ; 0)=\phi_{3} .
\end{aligned}
$$

Obviously, $D_{1}(\phi ; \tau)$ can be directly worked out as a constant. To solve the ODE for $D_{2}(\phi ; \tau)$, we transform the non-homogeneous initial condition into a homogeneous one with $\bar{D}_{2}(\phi ; \tau)=D_{2}(\phi ; \tau)-\phi_{2}$. We obtain

$\frac{d}{d \tau} \bar{D}_{2}=\frac{1}{2} \sigma^{2} \bar{D}_{2}^{2}+\left(\rho \sigma \phi_{1}+\sigma^{2} \phi_{2}-k\right) \bar{D}_{2}-\frac{1}{2}\left(\phi_{1}-\phi_{1}^{2}-2 \rho \sigma \phi_{1} \phi_{2}+2 k \phi_{2}-\sigma^{2} \phi_{2}^{2}\right), \bar{D}_{2}(\phi ; 0)=0$,

which is a Riccati equation with a homogeneous initial condition. The expression of $\bar{D}_{2}(\phi ; \tau)$ can then be derived as

$$
\bar{D}_{2}(\phi ; \tau)=\frac{d_{1}-\left(\rho \sigma \phi_{1}+\sigma^{2} \phi_{2}-k\right)}{\sigma^{2}} \cdot \frac{1-e^{d_{1} \tau}}{1-g_{1} e^{d_{1} \tau}} .
$$

Similarly, with $\bar{D}_{3}(\phi ; \tau)=D_{3}(\phi ; \tau)-\phi_{3}$, one could obtain

$$
\frac{d}{d \tau} \bar{D}_{3}=\frac{1}{2} \eta^{2} \bar{D}_{3}^{2}+\left(\eta^{2} \phi_{3}-\alpha\right) \bar{D}_{3}-\frac{1}{2}\left(2 \alpha \phi_{3}+2-2 \phi_{1}-\eta^{2} \phi_{3}^{2}\right), \bar{D}_{3}(\phi ; 0)=0,
$$

the solution of which can be found as

$$
\bar{D}_{3}(\phi ; \tau)=\frac{d_{2}-\left(\eta^{2} \phi_{3}-\alpha\right)}{\eta^{2}} \cdot \frac{1-e^{d_{2} \tau}}{1-g_{2} e^{d_{2} \tau}}
$$

With $D(\phi ; \tau)$ available, $C(\phi ; \tau)$ can now be worked out by integrating ${ }^{3}$ on both sides of its governing ODE with respect to $\tau$. This has completed the proof.

\footnotetext{
${ }^{3}$ When all the information of the Markov chain up to the expiry is given, $\theta_{X_{t}}$ and $\beta_{X_{t}}$ can then be treated as time-dependent functions instead of stochastic ones.
} 
With the conditional generalized moment generating function being analytically derived, as shown in Theorem 1, the remaining task is to compute the outer expectation contained in (3.6), which can now be expressed as

$$
\begin{aligned}
f\left(\phi, Y_{t}, X_{t}, t, T\right) & =E\left[m\left(\phi, Y_{t}, t, T \mid X_{T}\right) \mid X_{t}\right] \\
& =e^{D(\phi ; \tau) \cdot Y_{t}} E\left[e^{C(\phi ; \tau)} \mid X_{t}\right] .
\end{aligned}
$$

Following Elliott and Lian (2013), the expectation $E\left[e^{C(\phi ; \tau)} \mid X_{t}\right]$ can be calculated as

$$
\begin{aligned}
E\left[e^{C(\phi ; \tau)} \mid X_{t}\right] & =E\left[e^{\int_{t}^{T} k<\bar{\theta}, X_{s}>D_{2}(\phi ; T-s)+\alpha<\bar{\beta}, X_{s}>D_{3}(\phi ; T-s) d s}\right], \\
& =<e^{A^{T} \tau+B} X_{t}, I>,
\end{aligned}
$$

where $I=(1,1)^{T},\langle\cdot\rangle$ is the inner product for vectors, and $A$ is the transition rate matrix of the Markov chain $X_{t}$ and is defined as

$$
A=\left(\begin{array}{cc}
-\lambda_{12} & \lambda_{12} \\
\lambda_{21} & -\lambda_{21}
\end{array}\right) \text {. }
$$

$B$ can be expressed as

$$
B=\operatorname{diag}\left[k \bar{\theta} \int_{t}^{T} D_{2}(\phi ; T-s) d s+\alpha \bar{\beta} \int_{t}^{T} D_{3}(\phi ; T-s) d s\right]
$$

with diag[.] being the diagonal matrix constructed by putting the entries of the vector on the main diagonal. Therefore, the generalized moment generating function $f\left(\phi, Y_{t}, X_{t}, t, T\right)$ can finally be written as

$$
f\left(\phi, Y_{t}, X_{t}, t, T\right)=e^{D(\phi ; \tau) \cdot Y_{t}}<e^{A^{T} \tau+B} X_{t}, I>,
$$


where

$$
B=\left(\begin{array}{cc}
k \theta_{1} p_{1}(\phi ; \tau)+\alpha \beta_{1} p_{2}(\phi ; \tau) & 0 \\
0 & k \theta_{2} p_{1}(\phi ; \tau)+\alpha \beta_{2} p_{2}(\phi ; \tau)
\end{array}\right)
$$

with

$$
\begin{aligned}
& p_{1}(\phi ; \tau)=\frac{1}{\sigma^{2}}\left\{\left[d_{1}-\left(\rho \sigma \phi_{1}+\sigma^{2} \phi_{2}-k\right)\right] \tau-2 \ln \left(\frac{1-g_{1} e^{d_{1} \tau}}{1-g_{1}}\right)\right\}+\phi_{2} \tau \\
& p_{2}(\phi ; \tau)=\frac{1}{\eta^{2}}\left\{\left[d_{2}-\left(\eta^{2} \phi_{3}-\alpha\right)\right] \tau-2 \ln \left(\frac{1-g_{2} e^{d_{2} \tau}}{1-g_{2}}\right)\right\}+\phi_{3} \tau
\end{aligned}
$$

Till now, we have successfully derived a semi-analytical pricing formula for European call options, as presented in (3.3), with the expression of the generalized moment generating function shown in (3.13). In order to demonstrate the accuracy as well as various properties of the newly derived formula, numerical experiments are carried out in the next section to ensure the safe use of the new formula and provide some guidance for practical purposes.

\section{Numerical experiments and discussions}

In this section, we examine the influence of the newly introduced regime switching mechanics on the price of European options. Before we study the properties, our formula needs to be verified first to ensure that no algebraic errors are involved in the derivation process. In the following, unless otherwise stated, the mean-reverting speed of the volatility and that of the interest rate, i.e., $k$ and $\alpha$, are both set to 10 . The volatility of volatility and the volatility of the interest rate, i.e., $\sigma$ and $\eta$, are both set to 0.1 . The mean-reversion levels of the volatility in State 1 and State 2, i.e., $\theta_{1}$ and $\theta_{2}$, are set to 0.05 and 0.01 , respectively. The mean-reversion levels of the interest rate in both states, i.e., $\beta_{1}$ and $\beta_{2}$, are set to 0.05 and 0.01 , respectively. The correlation factor $\rho$ is assumed to be -0.8 , and the time to expiry $\tau$ is 1 year. Both of the current underlying price $S_{t}$ and the strike price $K$ are 
defaulted as 100. The spot values of the volatility and the interest rate, i.e., $v_{t}$ and $r_{t}$, are both set to 0.03 . The two transition rates, $\lambda_{12}$ and $\lambda_{21}$, both take the value of 10 , and the current state is assumed to be State 1.

Figure 1 shows the comparison between our prices (i.e., option prices calculated from our formula) and the Monte Carlo prices (i.e., option prices obtained through Monte Carlo simulation). Depicted in Figure 1(a) are the two option prices against underlying prices. From this figure, it is clear that the two prices are point-wisely close to each other for every underlying value. The relative difference between our price and the Monte Carlo price, as further shown in Figure 1(b), is less than 0.9\%. This implies that our newly derived pricing formula is correct and can be safely used in practice.

With confidence in our newly derived formula, we can now study the influence of regime switching on the option price. With both transition rates being assumed to be the same, option prices $^{4}$ are plotted against the transition rate in Figure 2. As expected, the option price under the Heston-CIR model remains constant when the transition rate is varying, because the Heston-CIR model is independent of the transition rate. Furthermore, the option price under our regime switching Heston-CIR model degenerates to the Heston-CIR price when the transition rate is equal to 0 . This is not surprising due to the fact that there is no actual regime switching in our model when the transition rate is 0 . In addition, when the mean-reversion levels of both the volatility and the interest rate in State 1 are lower than the corresponding ones in State 2, our price is a monotonic increasing function of the transition rate. A completely opposite trend can be observed when those values in State 1 are higher than the corresponding ones in State 2. This could be explained by the fact that a higher transition rate means a larger chance for the mean- reversion levels to jump from State 1 to State 2, leading to a larger opportunity in getting a higher/lower mean-reversion level as well as higher/lower volatility and interest rate.

\footnotetext{
${ }^{4}$ For comparison purposes, the Heston-CIR price are calculated with the mean-reversion levels of the volatility and the interest rate being the same as the corresponding ones used in State 1 of the regime switching Heston-CIR model because the current state is assumed to be State 1.
} 


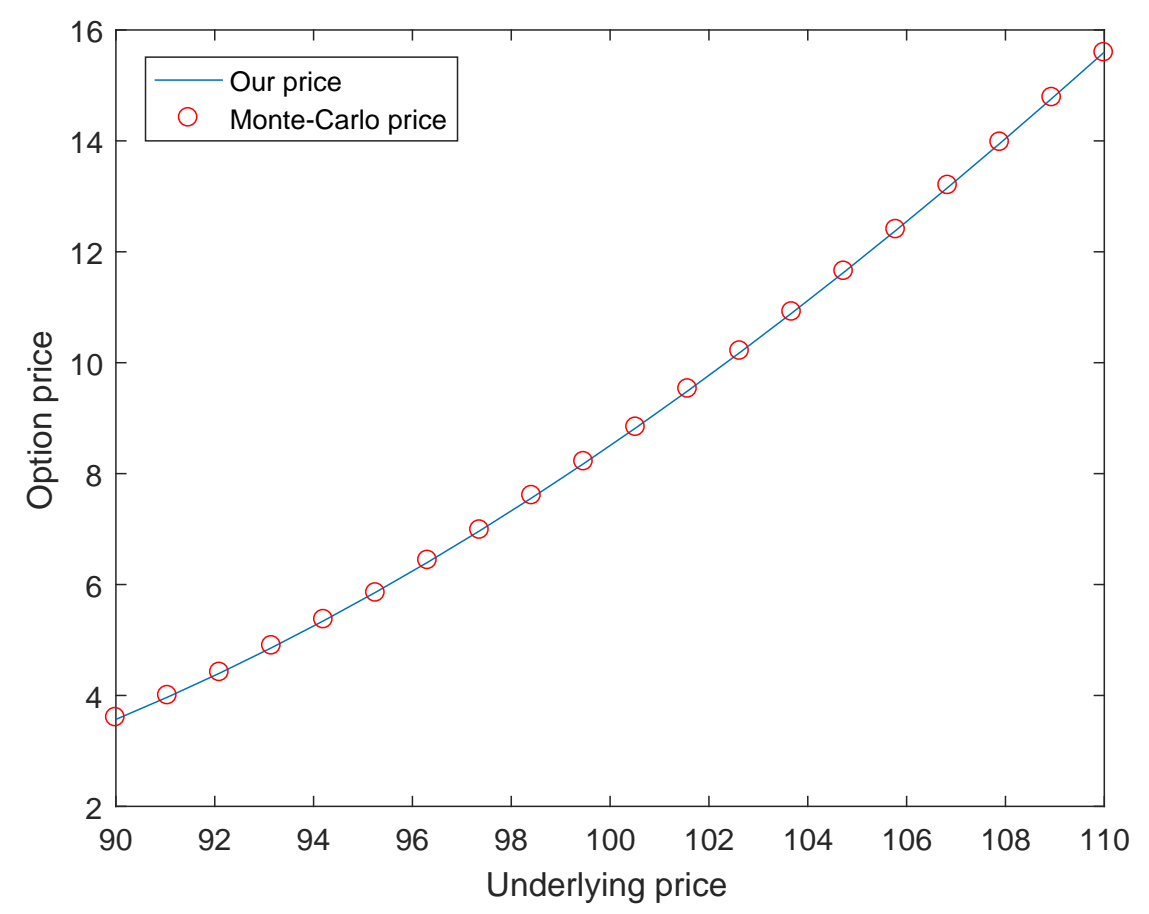

(a) Our price vs Monte-Carlo price.

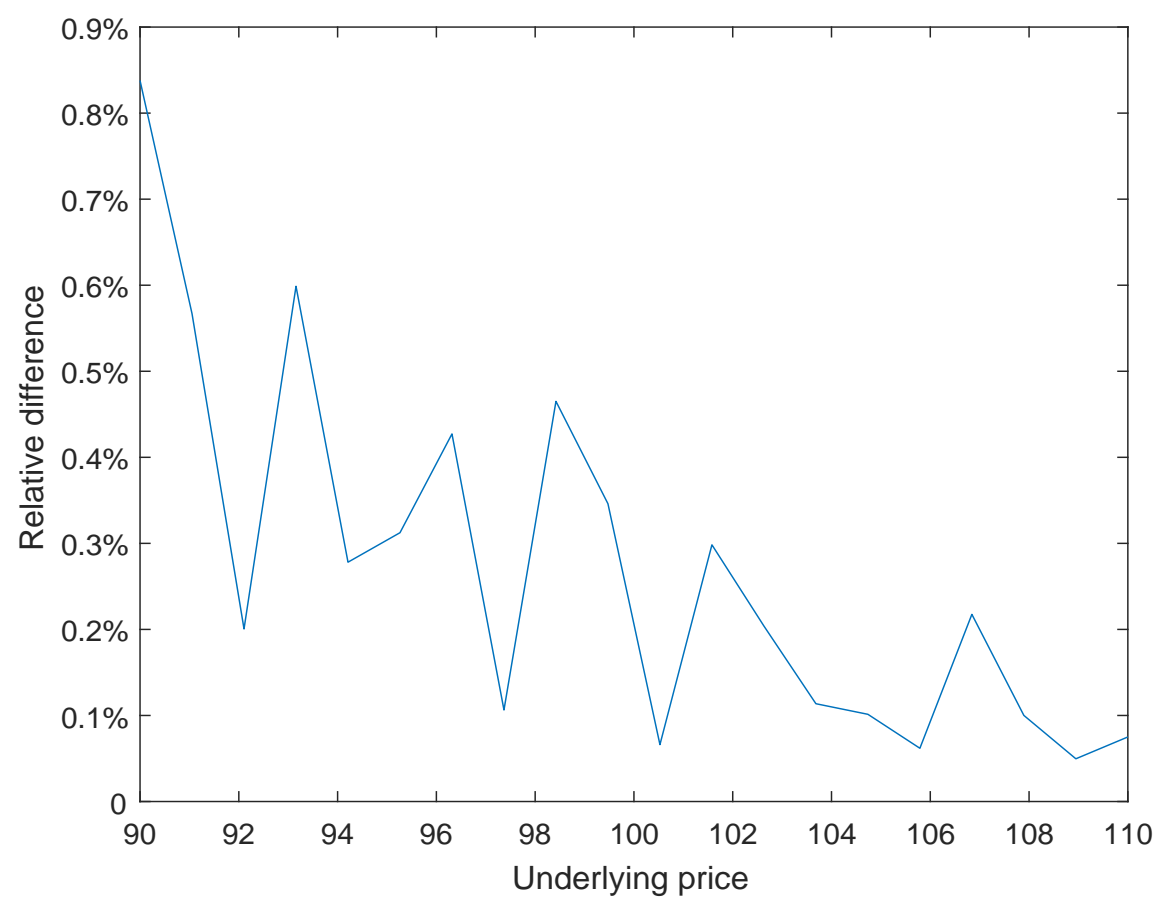

(b) The relative difference between our price and Monte-Carlo price.

Figure 1: The comparison of option prices calculated with our formula and those obtained through Monte Carlo simulation. 


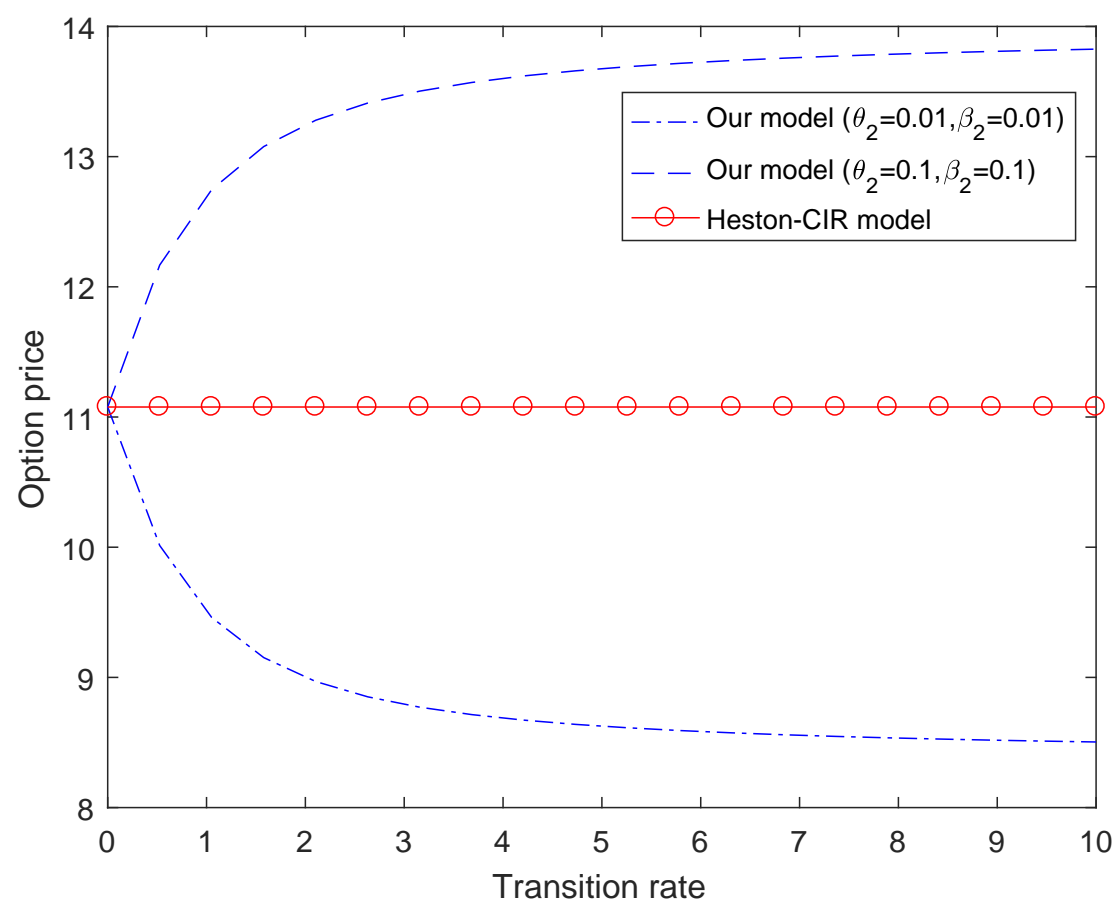

Figure 2: Our price vs Heston-CIR price with respect to different transition rates.

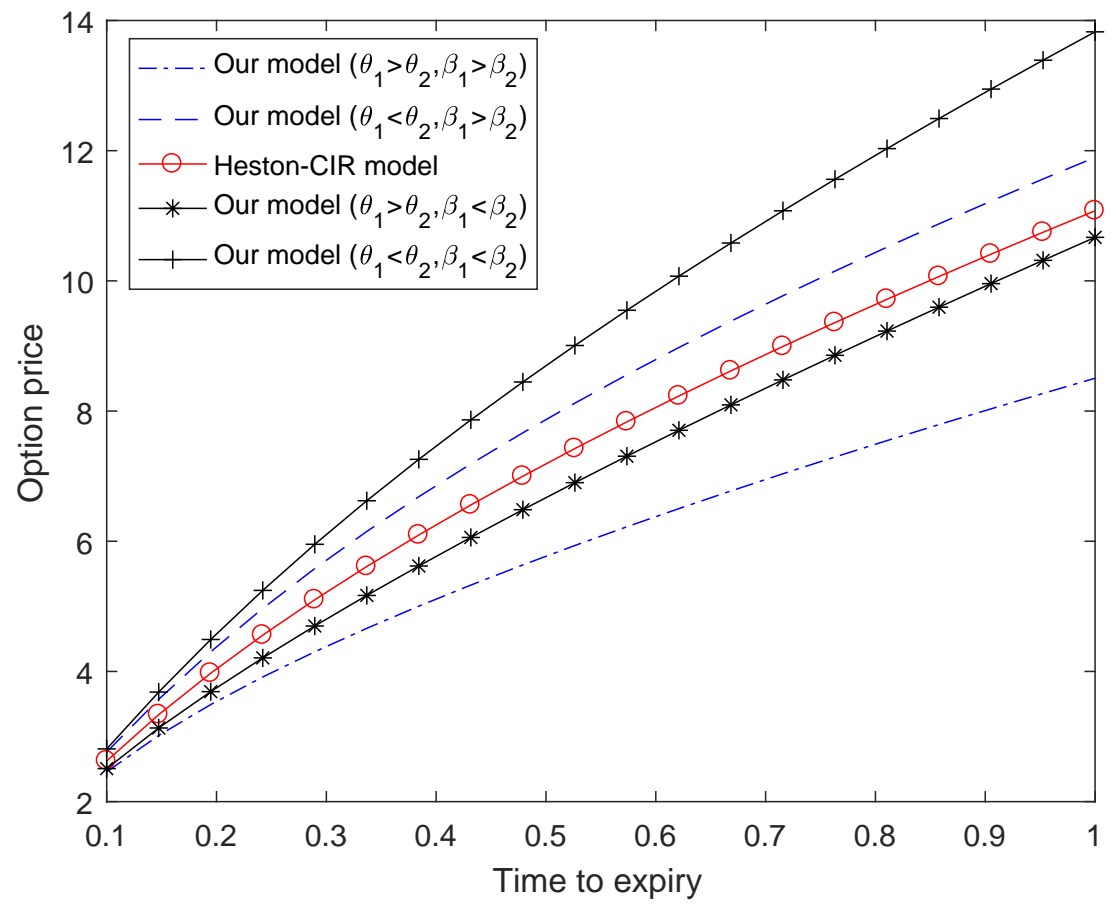

Figure 3: Our price vs Heston-CIR price with respect to different time to expiry. 
To further investigate the effect of the mean-reversion levels of the volatility and the interest rate on the option price, we keep $\theta_{1}$ and $\beta_{1}$ unchanged as 0.05 , while let both $\theta_{2}$ and $\beta_{2}$ vary between 0.01 and 0.1 . The option prices as a function of the time to expiry are provided in Fig 3. From this figure, one could observe that when the mean-reversion levels

of the volatility and the interest rate in State 2 are higher (lower) than the corresponding values in State 1, our price is larger (smaller) than the Heston-CIR price. Moreover, it can also be observed that if the mean-reversion level of the volatility in State 2 is higher (lower) than that in State 1 while the mean-reversion level of the interest rate in State 2 is lower (higher) than that in State 1, our price is still greater (smaller) than the Heston-CIR price, though it does decrease (increase) a bit. This suggests that the mean-reversion level of the volatility has a greater impact on option prices than the interest rate does.

Of course, the comparison made between our model and the Heston-CIR model in this section is based on the fact that the corresponding parameters in both models are kept the same. However, it is widely acknowledged that any mathematical model needs to go through a model calibration process, in which model parameters will be determined from real market data and the corresponding parameters may not be the same after this process. Thus, we are still not sure the performance of our model in real markets, and this will be discussed in the next section.

\section{Empirical studies}

In this section, the performance of our model will be assessed through empirical studies by taking the Heston-CIR model as a benchmark. The data set together with the application of several appropriate filters is firstly introduced. The approach for conducting model calibration is then described. The empirical results are finally presented and analyzed. 


\subsection{Data description}

This preliminary empirical study is based on European call options written on the S\&P 500 Index ranging from from Jan 2012 to Mar 2012. As one may be aware, there are two prices for one option in practice, i.e., bid and ask prices, and a common approach is to use the average of the two as the price of the option for model calibration. Moreover, sample noises contained in the raw data set should also be eliminated prior to empirical studies to avoid possible mis-leading conclusions.

First of all, a common practice in model calibration is that only Wednesday options data are used for the parameter estimation ${ }^{5}$, because Wednesday is least likely to be holidays in a week and less likely to be affected by the "day-of-the-week" effect (Bakshi et al., 1997; Christoffersen et al., 2006). Secondly, we have also discarded the options with the time to expiry being less than 30 days and more than 90 days, because the options with low time to expiry tend to have small time values and their prices could be very volatile, while the options with large time to expiry usually have liquidity problems due to their high trading premium (Le, 2015). Thirdly, very deep in-the-money and very deep out-of-the-money options also have liquidity problems (Shu and Zhang, 2004), and should also be removed. Therefore, we have also excluded the options with the absolute moneyness ${ }^{6}$ being higher than $10 \%$.

Having applied all these filters, we have also chosen the three-month daily U.S. Treasury Bill Rate as a proxy of the initial level of the risk-free interest rate (Benjamin et al., 2007; Shu and Zhang, 2004). Will all these prior steps being taken, we are now ready to proceed to the parameter estimation stage, the details of which are presented in the next subsection.

\footnotetext{
${ }^{5}$ Thursday data will also be adopted, but for assessing the out-of-sample performance.

${ }^{6}$ The absolute moneyness is defined as the absolute relative difference between the underlying price $S$ and strike $K$, or equivalently, $\left|\frac{S-K}{K}\right|$.
} 


\subsection{Parameter estimation}

A necessary step before conducting parameter estimation for any model is always to figure out the model parameters that need to be determined from real market data. In particular, the Heston-CIR model, as the benchmark, can be specified as

$$
\begin{aligned}
\frac{d S_{t}}{S_{t}} & =r_{t} d t+\sqrt{v_{t}} d W_{t}^{S} \\
d v_{t} & =k\left(\theta-v_{t}\right) d t+\sigma \sqrt{v_{t}} d W_{t}^{v} \\
d r_{t} & =\alpha\left(\beta-r_{t}\right) d t+\eta \sqrt{r_{t}} d W_{t}^{r}
\end{aligned}
$$

From this, one can clearly observe that there are eight model parameters in the HestonCIR model, including four parameters associated with the volatility process, $k, \theta, \sigma, v_{0}$, three parameters associated with the interest rate $\alpha, \beta, \eta$, and one correlation factor $\rho$. On the other hand, in comparison to the Heston-CIR model, four additional parameters are incorporated into our model due to the introduction of regime switching. They are the second mean-reversion level of volatility and interest rate, $\theta_{2}, \beta_{2}$, and two transition rates $\lambda_{12}$ and $\lambda_{21}$.

With the target model parameters in hand, a natural question is how to extract the values of those parameters from option data available in real markets such that the produced model prices are closest to the market prices considered. This implies that we need to find an appropriate measurement for the distance. Following Christoffersen and Jacobs (2004); Lim and Zhi (2002), the measurement we choose here is the so-called dollar mean-squared error (MSE) defined as

$$
M S E=\frac{1}{N} \sum_{i=1}^{N}\left[C^{\text {Market }}-C^{\text {Model }}\right]^{2}
$$

where $C^{\text {Market }}$ and $C^{\text {Model }}$ are the market price of an option and the price of the same option calculated from our formula, respectively, and $N$ is the total number of observations selected in a single estimation. 
Clearly, the model calibration problem has now been formulated as a minimization problem, i.e., finding an "optimal" set of parameters that can minimize the MSE defined in (5.1). Therefore, it is vital to choose a good optimization technique. We remark that in the current work, the local minimization technique is not adopted, although it might be easy and fast to implement. This technique depends heavily on the initial guess, without an appropriate choice of which it will probably end up with a local minimum since our objective function (5.1) is not necessarily convex and thus there exist several local minima. Therefore, in the current work, the global optimization approaches are preferred because they can skip local minima to ensure the attainability of the global minimum.

Of all the global optimization approaches, the Simulated Annealing (SA) (Kirkpatrick et al., 1983) is well known because it is easy to program and only has few parameters requiring tuning, and most importantly, it theoretically guarantees the convergence to the global minimum. However, the slow speed of convergence hinders the potential application of this great technique to solve practical problems. To effectively determine model parameters, in the following, we adopt the Adaptive Simulated Annealing (ASA), which is a well-known variation of SA (Ingber, 1989), and is designed to find the best global fit of a non-linear constrained non-convex cost function over a D-dimensional space (Ingber et al., 2012). The ASA is not only possessing all the advantages of the SA, but also more efficient and less sensitive to user defined parameters than the SA does. Due to its superiority, the ASA has already been widely applied to various areas, including the calibration of option pricing models (Poklewski-Koziell, 2012; Mikhailov and Nögel, 2004).

Another main advantage of the ASA is that it can be implemented through the opensource code provided in Ingber (2018), as a result of which its flexibility and powerfulness can be enhanced from the feedback produced by different users. By applying the ASA to minimize the MSE defined in (5.1) with the data described in the previous subsection, the estimated daily-averaged parameters for both models are reported in Table 1.

Once parameters have been determined from real market data, we shall proceed to 
Table 1: Estimated parameters.

\begin{tabular}{c|cc}
\hline Parameters & Our model & Heston-CIR model \\
\hline$k$ & 6.4192 & 7.9405 \\
$\theta_{1}(\theta)$ & 0.1312 & 0.1235 \\
$\theta_{2}$ & 0.1488 & \\
$\sigma$ & 0.4911 & 0.5212 \\
$\alpha$ & 4.1459 & 4.4712 \\
$\beta_{1}(\beta)$ & 0.1648 & 0.3663 \\
$\beta_{2}$ & 0.4618 & \\
$\eta$ & 3.3442 & 3.3262 \\
$\rho$ & -0.5309 & -0.5714 \\
$v_{0}$ & 0.0185 & 0.0194 \\
$\lambda_{12}$ & 5.5336 & \\
$\lambda_{21}$ & 2.5783 & \\
\hline
\end{tabular}

assessing the performance of both models. This issue will be illustrated in the next subsection.

\subsection{Empirical results}

The model performance can be assessed from two different aspects. One is the "in-sample error", defined as the remaining error between market and model prices after model calibration. The other one is the "out-of-sample error", which is referred to as the "prediction" error calculated with market prices of another data set that is not used for model calibration and the corresponding model prices produced by the parameters determined from model calibration. Table 2 below exhibits both of in- and out-of-sample errors of the two models.

Table 2: In- and out-of-sample errors for the two models

\begin{tabular}{c|cc}
\hline Error & In-sample & out-of-sample \\
\hline Our model & 0.0522 & 1.2658 \\
Heston-CIR model & 0.0644 & 1.5805 \\
\hline
\end{tabular}

The magnitude of both in- and out-of-sample errors of the two models shown in Table 2 clearly reveals that our model with regime switching greatly outperforms the HestonCIR model without regime switching. In specific, from the in-sample point of view, the 
daily averaged MSE for our model is 0.0522 , which is approximately $81 \%$ of that produced by the Heston-CIR model. A similar pattern can also be observed in the out-of-sample errors, with the corresponding MSE of our model being around $80 \%$ of that under the Heston-CIR model. The consistency in the extent of improvement occurred in both inand out-of-sample errors demonstrates that the introduction of regime switching is vital in achieving a better data fitness, and thus our model serves as a better choice than the Heston-CIR model, at least for the chosen data set.

Table 3: Out-of-sample errors according to moneyness

\begin{tabular}{c|ccc}
\hline Moneyness & out-of-money & at-the-money & in-the-money \\
\hline Our model & 0.3609 & 1.9613 & 1.2096 \\
Heston-CIR model & 0.3438 & 2.4585 & 1.3257 \\
\hline
\end{tabular}

Another issue with common interest is the behavior of both models across different moneyness. Thus, the out-of-sample errors produced by out-of-money, at-the-money and in-the-money options, which are classified according to $0.90<S / K<0.97,0.97 \leq S / K \leq$ 1.03 and $1.03<S / K<1.10$, respectively, are shown in Table 3. Although our model provides slightly worse performance for out-of-money options, there is a great improvement in the out-of sample errors associated with both at-the-money and in-the-money options, with our model showing about $20 \%$ and $10 \%$ less errors than the Heston-CIR model. As a result, we can confidently conclude that our model can of course act as a good competitor to the Heston-CIR model in real markets.

\section{Conclusion}

This paper investigates the pricing of European options under the Heston-CIR model with regime switching mechanics. We derive a semi-analytical formula for European options under this complicated model after solving the generalized moment generating function of the underlying price, volatility and interest rate. Numerical experiments are also carried out to show the accuracy of the formula and various properties of option prices under 
this particular model, the results of which suggest that the introduction of the regime switching has a significant impact on the option price. Finally, through an empirical study, our model demonstrates a better performance than the Heston-CIR model for the test data sets, implying that our model can at least be served as a good competitor of the Heston-CIR model in practice.

\section{Acknowledgments}

This work is supported by National Natural Science Foundation of China No. 11601189, and Natural Science Foundation of Jiangsu Province No. BK20160156.

\section{References}

Abudy, M. and Izhakian, Y. (2013), 'Pricing stock options with stochastic interest rate', International Journal of Portfolio Analysis and Management 1(3), 250-277.

Bakshi, G., Cao, C. and Chen, Z. (1997), 'Empirical performance of alternative option pricing models', The Journal of Finance 52(5), 2003-2049.

Beckers, S. (1983), 'Variances of security price returns based on high, low, and closing prices', Journal of Business pp. 97-112.

Benjamin, M. A., Hinnant, H. O., Shigeno, T. T. and Olmstead, D. N. (2007), 'Multi-sensor fusion'. US Patent 7,283,904.

Black, F. and Scholes, M. (1973), 'The pricing of options and corporate liabilities', The Journal of Political Economy pp. 637-654.

Buffington, J. and Elliott, R. J. (2002), 'American options with regime switching', International Journal of Theoretical and Applied Finance 5(05), 497-514. 
Cao, J., Roslan, Teh Raihana Nazirah, R. and Zhang, W. (2018), 'Pricing variance swaps in a hybrid model of stochastic volatility and interest rate with regime-switching', Methodology and Computing in Applied Probability pp. 1-21.

Christoffersen, P. and Jacobs, K. (2004), 'Which GARCH model for option valuation?', Management Science 50(9), 1204-1221.

Christoffersen, P., Jacobs, K. and Mimouni, K. (2006), 'An empirical comparison of affine and non-affine models for equity index options', Manuscript, Springer .

Duffie, D., Pan, J. and Singleton, K. (2000), 'Transform analysis and asset pricing for affine jump-diffusions', Econometrica 68(6), 1343-1376.

Dumas, B., Fleming, J. and Whaley, R. E. (1998), 'Implied volatility functions: Empirical tests', The Journal of Finance 53(6), 2059-2106.

Elliott, R. J. and Lian, G.-H. (2013), 'Pricing variance and volatility swaps in a stochastic volatility model with regime switching: discrete observations case', Quantitative Finance 13(5), 687-698.

Eraker, B. (2004), 'Do stock prices and volatility jump? reconciling evidence from spot and option prices', The Journal of Finance 59(3), 1367-1404.

Forde, M. and Jacquier, A. (2010), 'Robust approximations for pricing Asian options and volatility swaps under stochastic volatility', Applied Mathematical Finance 17(3), 241259.

Grzelak, L. A. and Oosterlee, C. W. (2011), 'On the Heston model with stochastic interest rates', SIAM Journal on Financial Mathematics 2(1), 255-286.

Hamilton, J. D. (1990), 'Analysis of time series subject to changes in regime', Journal of Econometrics 45(1), 39-70. 
He, X.-J. and Zhu, S.-P. (2016a), 'An analytical approximation formula for European option pricing under a new stochastic volatility model with regime-switching', Journal of Economic Dynamics and Control 71, 77-85.

He, X.-J. and Zhu, S.-P. (2016b), 'Pricing European options with stochastic volatility under the minimal entropy martingale measure', European Journal of Applied Mathematics $\mathbf{2 7}(02), 233-247$.

He, X.-J. and Zhu, S.-P. (2017), 'How should a local regime-switching model be calibrated?', Journal of Economic Dynamics and Control 78, 149-163.

He, X.-J. and Zhu, S.-P. (2018a), 'A closed-form pricing formula for European options under the Heston model with stochastic interest rate', Journal of Computational and Applied Mathematics 335, 323-333.

He, X.-J. and Zhu, S.-P. (2018b), 'On full calibration of hybrid local volatility and regimeswitching models', Journal of Futures Markets 38(5), 586-606.

Heston, S. L. (1993), 'A closed-form solution for options with stochastic volatility with applications to bond and currency options', Review of Financial Studies 6(2), 327-343.

Hull, J. and White, A. (1987), 'The pricing of options on assets with stochastic volatilities', The Journal of Finance 42(2), 281-300.

Ingber, L. (1989), 'Very fast simulated re-annealing', Mathematical and Computer Modelling 12(8), 967-973.

Ingber, L. (2018), 'Home page of lester ingber', https://www.ingber.com .

Ingber, L., Petraglia, A., Petraglia, M. R., Machado, M. A. S. et al. (2012), Adaptive simulated annealing, in 'Stochastic global optimization and its applications with fuzzy adaptive simulated annealing', Springer, pp. 33-62. 
Johnson, H. and Shanno, D. (1987), 'Option pricing when the variance is changing', Journal of Financial and Quantitative Analysis 22(02), 143-151.

Kirkpatrick, S., Gelatt, C. D., Vecchi, M. P. et al. (1983), 'Optimization by simulated annealing', Science 220(4598), 671-680.

Le, A. (2015), 'Separating the components of default risk: A derivative-based approach', The Quarterly Journal of Finance 5(01), 1550005.

Lim, K. G. and Zhi, D. (2002), 'Pricing options using implied trees: Evidence from FTSE100 options', Journal of Futures Markets 22(7), 601-626.

Mikhailov, S. and Nögel, U. (2004), 'Hestons stochastic volatility model: Implementation, calibration and some extensions', Wilmott Magazine p. 7479.

Poklewski-Koziell, W. (2012), Stochastic volatility models: Calibration, pricing and hedging, PhD thesis.

Scott, L. O. (1987), 'Option pricing when the variance changes randomly: Theory, estimation, and an application', Journal of Financial and Quantitative Analysis 22(04), 419438.

Shu, J. and Zhang, J. E. (2004), 'Pricing S\&P 500 index options under stochastic volatility with the indirect inference method', Journal of Derivatives Accounting 1(2), 1-16.

Stein, E. M. and Stein, J. C. (1991), 'Stock price distributions with stochastic volatility: An analytic approach', Review of Financial Studies 4(4), 727-752.

Vo, M. T. (2009), 'Regime-switching stochastic volatility: Evidence from the crude oil market', Energy Economics 31(5), 779-788.

Wiggins, J. B. (1987), 'Option values under stochastic volatility: Theory and empirical estimates', Journal of Financial Economics 19(2), 351-372. 
Zhu, S.-P. and He, X.-J. (2017), 'On the convergence of He and Zhu's new series solution for pricing options with the Heston model', Acta Mathematica Universitatis Comenianae 86(2), 321-327. 